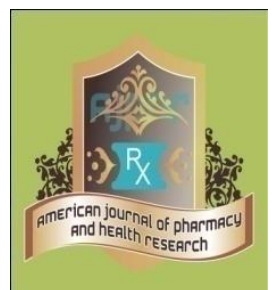

Review Article

AMERICAN JOURNAL OF PHARMACY AND HEALTH RESEARCH

www.ajphr.com

2021, Volume 9, Issue 10

ISSN: 2321-3647(online)

\title{
A Review on Chemistry of Anti-malarials
}

\section{P. Margret Chandira ${ }^{*}{ }^{1}$ CH.NVS Mastanrao ${ }^{2}$, B.S.Venkateswarlu}

1. Professor Department of pharmaceutics, Vinayaka Mission's Research Foundation, Sankari

Main Road, Ariyanur, Tamil Nadu -636308.

2. Research Scholar, Vinayaka Missions Research Foundation, Salem, Tamil nadu, India-

636008. Assistant professor in Nalanda Institute of Pharmaceutical sciences, Kantepudi,

Sattenapalli, Guntur, 522403

3. Professor, Vinayaka Mission's Research Foundation, Sankari Main Road, Ariyanur, Tamil

Nadu -636308

\begin{abstract}
Malaria remains an important public health concern in countries where transmission occurs regularly, as well as in temperate areas and it is a major global health problem, with an estimated 500 million clinical cases occurring annually. Malaria is a complex disease that varies widely in epidemiology and clinical manifestation in different parts of the world. The most prevalent and dangerous type of malaria is caused by Plasmodium falciparum. P. vivax is a common cause of malaria in Latin America, Asia, and Oceania, but not Africa. P. malaria and P. ovale are much less common. Antimalarials are used in three different ways: prophylaxis, treatment of falciparum malaria, and treatment of non-falciparum malaria. Prophylactic antimalarials are used almost exclusively by travelers from developed countries who are visiting malaria-endemic countries.
\end{abstract}

Keywords: Malaria, antimalarial drugs, plasmodium. 


\section{INTRODUCTION}

Malaria is a complex disease that varies widely in epidemiology and clinical manifestation in different parts of the world. This variability is the result of factors such as the species of malaria parasites that occur in a given area, their susceptibility to commonly used or available antimalarial drugs, the distribution and efficiency of mosquito vectors, climate and other environmental conditions and the behavior and level of acquired immunity of the exposed human populations. In particular, young children, pregnant women, and non-immune visitors to malarious areas are at greatest risk of severe or fatal illness. Many malaria control strategies exist, but none are appropriate and affordable in all contexts. Malaria control and prevention efforts need to be designed for the specific environment in which they will be used and need to take into account the local epidemiology of malaria and the level of available resources and political will.

Malaria is caused by infection with a single-cell parasite, Plasmodium. Four Plasmodium spp. cause malaria in human beings: Plasmodium falciparum, P. vivax, P. ovale, and P. malariae. P. falciparum is the most important because it accounts for the majority of infections and causes the most severe symptoms. Antimalarials are used in three different ways: prophylaxis, treatment of falciparum malaria, and treatment of non-falciparum malaria. Prophylactic antimalarials are used almost exclusively by travelers from developed countries who are visiting malaria endemic countries. Treatment protocols for falciparum malaria vary, depending on the severity of the disease; fast-acting, parenteral drugs are best for severe, life threatening disease. In addition, treatment protocols for falciparum malaria vary geographically and depend on the resistance profiles for strains in particular regions. Non-falciparum malarias, in contrast, rarely are drug resistant. In addition, P. vivax and P. ovale have dormant liver stages that can cause relapses months to years after an infection is cleared, so they need to be treated with an additional agent that can clear this stage. The antimalarials in common use come from following classes of compounds: the quinolines (chloroquine, quinine, mefloquine, amodiaquine, primaquine), the antifolate (pyrimethamine, proguanil and sulfadoxine), the artmisinin derivatives (artemisinin, artesunate, artemether, arteether) and hydroxynaphthaquinones (atovaquine).

This review looks at the drugs in common use and their treatment regimens, pharmacokinetic properties, mechanism of action and resistance, a status of resistance.

Drug resistance has been implicated in the spread of malaria to new areas and re-emergence of malaria in areas where the disease had been eradicated. Drug resistance has also played a significant role in the occurrence and severity of epidemics in some parts of the world. 
Population movement has introduced resistant parasites to areas previously free of drug resistance. The economics of developing new pharmaceuticals for tropical diseases, including malaria, are such that there is a great disparity between the public health importance of the disease and the amount of resources invested in developing new cures $(1,2)$. This disparity comes at a time when malaria parasites have demonstrated some level of resistance to almost every antimalarial drug currently available, significantly increasing the cost and complexity of achieving parasitological cure. The purpose of this review is to describe the state of knowledge regarding drug- resistant malaria and to outline the current thinking regarding strategies to limit the advent, spread, and intensification of drug resistant malaria.

\section{Causative agents:}

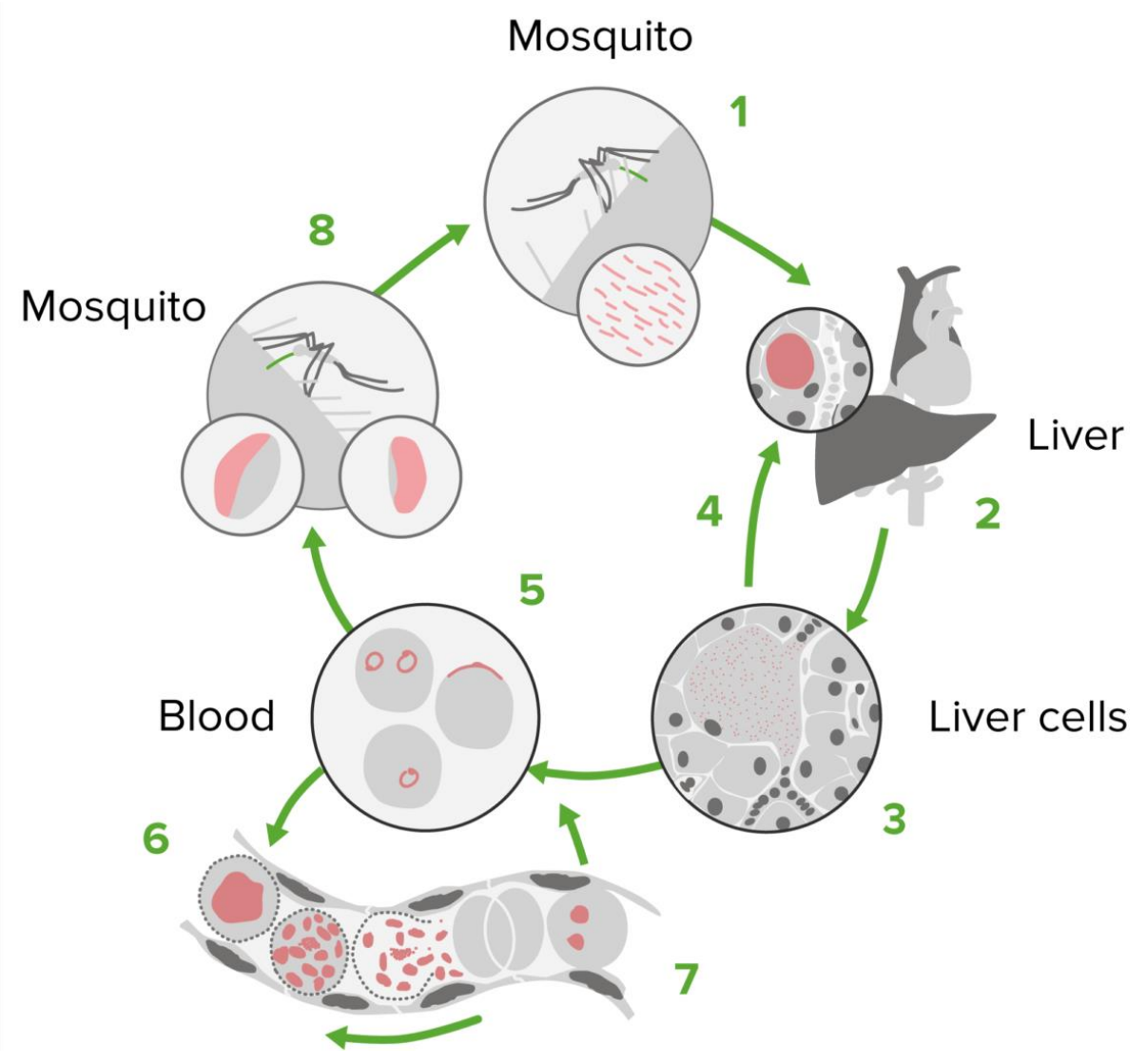

Figure 1: Life cycle of plasmodium species

In humans, malaria infection is caused by one or more of four species of intracellular protozoan parasite. Plasmodium falciparum, P. vivax, P. ovale, and P. malariae differ in geographical distribution, microscopic appearance, clinical features (periodicity of infection, potential for severe disease, and ability to cause relapses), and potential for development of resistance to antimalarial drugs. To date, drug resistance has only been documented in two of the four species, P. falciparum and P. vivax

\section{Drugs available for treatment of malaria:}


There are only a limited number of drugs which can be used to treat or prevent malaria. The most widely used are quinine and its derivatives and antifolate combination drugs.

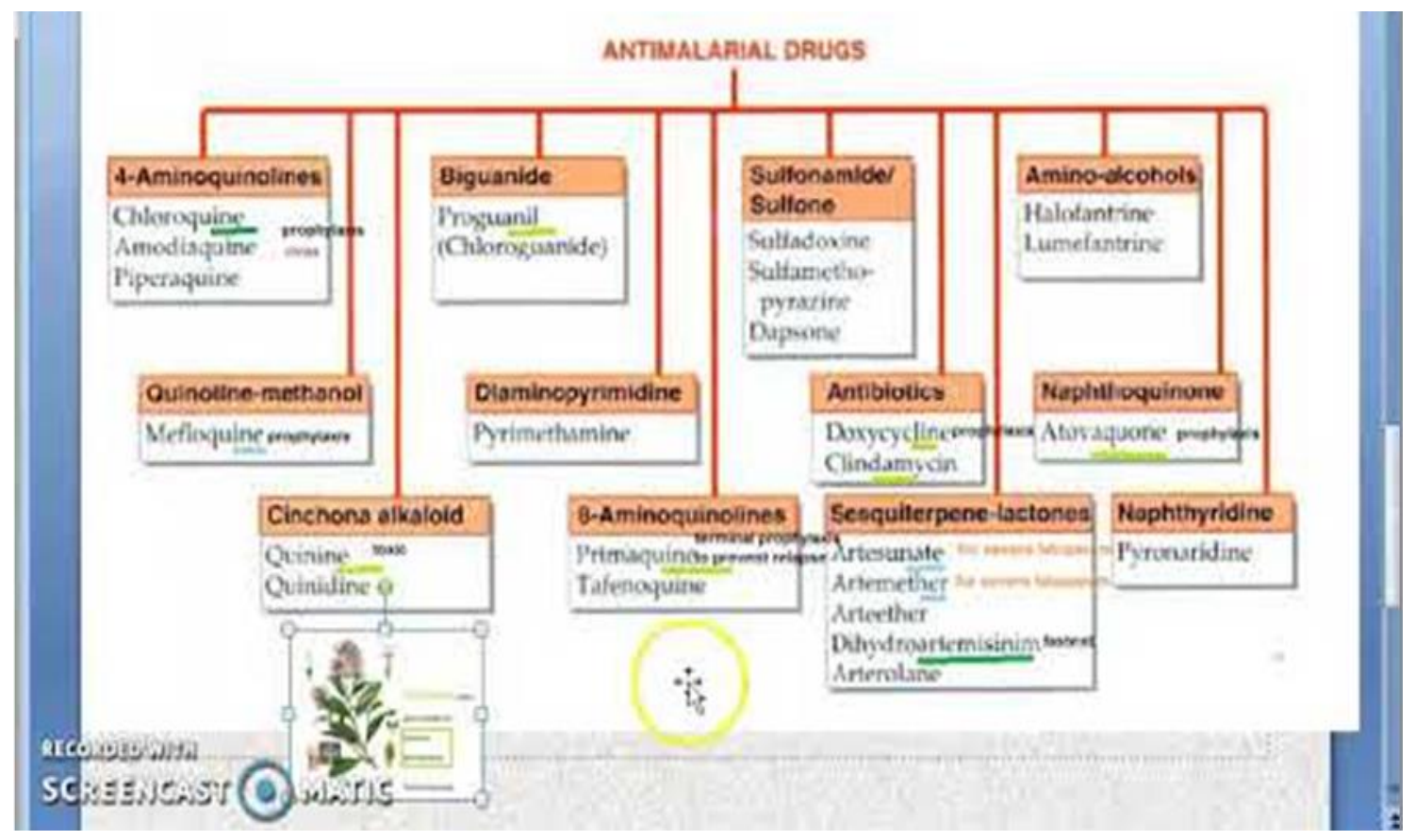

\section{Antimalarials are classified}

1. According to anti-malarial activity

2. According to the structure

\section{According to anti malarial activity:}

1. Tissue schizonticides for causal prophylaxis:

These drugs act on the primary tissue forms of the plasmodia which after growth within the liver, initiate the erythrocytic stage. By blocking this stage, further development of the infection can be theoretically prevented. Pyrimethamine and Primaquine have this activity. However since it is impossible to predict the infection before clinical symptoms begins; this mode of therapy is more theoretical than practical.

\section{Tissue schizonticides for preventing relapse}

These drugs act on the hypnozoites of P. vivax and P. ovale in the liver that cause relapse of symptoms on reactivation. Primaquine is the prototype drug; pyrimethamine also has such activity.

\section{Blood schizonticides:}

These drugs act on the blood forms of the parasite and thereby terminate clinical attacks of malaria. These are the most important drugs in anti-malarial chemotherapy. These include chloroquine, quinine, mefloquine, halofantrine, pyrimethamine, sulfadoxine, sulfones, tetracycline's etc. 


\section{Gameto cytocides:}

These drugs destroy the sexual forms of the parasite in the blood and thereby prevent transmission of the infection to the mosquito. Chloroquine and quinine have Gametocytocides activity against P. vivax and P. malariae, but not against P. falciparum. Primaquine has gametocytocidal activity against all plasmodia, including P. falciparum.

\section{Sporontocides:}

These drugs prevent the development of oocysts in the mosquito and thus ablate the transmission. Primaquine and chloroguanide have this action. Thus in effect, treatment of malaria would include a blood schizonticide, a gametocytocide and a tissue schizonticide (in case of P. vivax and P. ovale). A combination of chloroquine and primaquine is thus needed in ALL cases of malaria

\section{According to the structure:}

1. Aryl amino alcohols: Quinine, guanidine (cinchona alkaloids), mefloquine, halofantrine.

2. 4-aminoquinolines: Chloroquine, amodiaquine.

3. Folate synthesis inhibitors:

4. Type 1 competitive inhibitors of dihydropteroate syntheses - sulphones, sulphonamides;

5. Type 2 inhibit dihydrofolate reductase - biguanides like proguanil and chloroproguanil; di amino pyrimidine like pyrimethamine

6. 8-aminoquinolines: Primaquine

7. Antimicrobials: Tetracycline, doxycycline, clindamycin, azithromycin, fluoroquinolones

8. Peroxides: Artemisinin derivatives and analogues - artemether, arteether, artesunate, artelinic acid

9. Naphtho quinines: Atovaquone 8. Iron chelating agents: Desferrioxamine

\section{Mechanism of action of Antimalarials:}


Mechanism of action of quinoline drugs

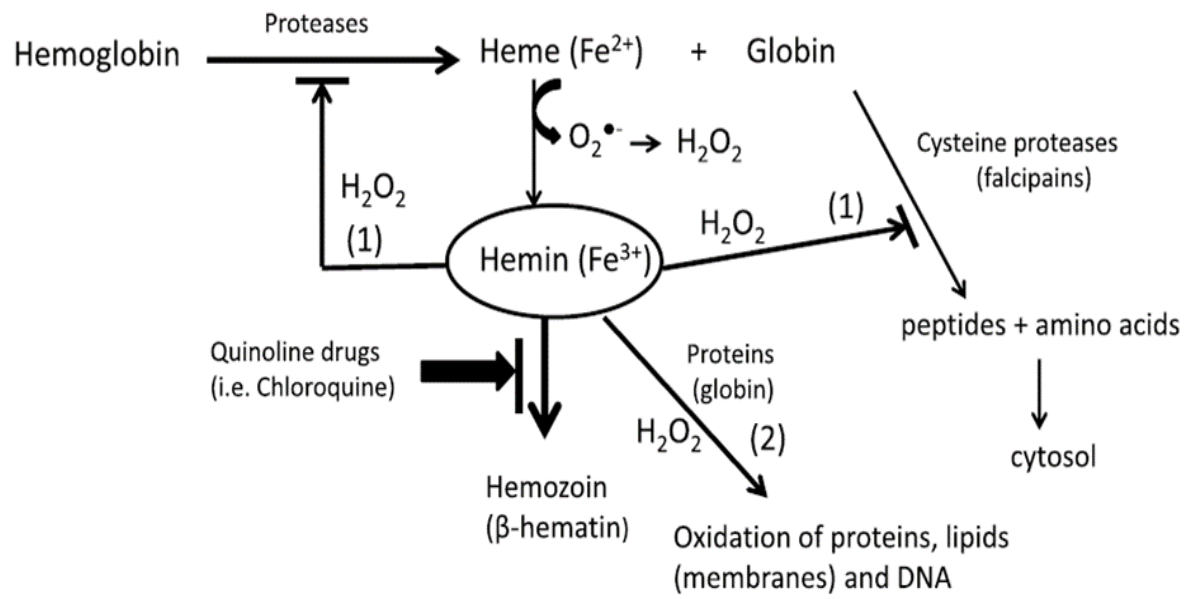

Mechanism of action of quinoline drugs inhibiting hemozoin in the digestive vacuole of Plasmodium inside erythrocytes or in other organisms that use hemoglobin as food. This inhibition enhances the levels of free hemin which promote peroxidase reactions with $\mathrm{H} 2 \mathrm{O} 2$ arising from heme (Fe2+) oxidation. Hemin and

peroxidase reactions inhibit cysteine proteases needed for degradation of proteins used for parasite growth (1), and may also oxidize proteins (enzymes), lipids and DNA resulting in parasite damage (2).

Herraiz T, Guillén H, González-Peña D, Arán VJ. Antimalarial Quinoline Drugs Inhibit B-Hematin and Increase Free Hemin Catalyzing Peroxidative Reactions and Inhibition of Cysteine Proteases. Sci Rep. 2019 Oct 28:9(1):15398. PMID: 31659177.

\section{Chemistry of antimalarials:}

\section{Artemisinin:}

- Artemisinin is a natural product that can be extracted from the leaves of Artemisia annua

- Formula : C15H22O5

- Molar mass : $282.332 \mathrm{~g} / \mathrm{mol}$

- Density : $1.24 \pm 0.1 \mathrm{~g} / \mathrm{cm} 3$

- M.P : 152 to $157^{\circ} \mathrm{C}\left(306\right.$ to $\left.315^{\circ} \mathrm{F}\right)$

- Artemisinin The therapeutic utility of the lead itself is limited by poor physicochemical properties such as poor oil \& water solubility Artemisinin

- Artemisinin serve as a lead compound for the development of new antimalarials with improved properties - The lactone group can be reduced \& form dihydroartemisinin which is used to prepare semi synthetic prodrug that are more water \& oil soluble

- The hydroxyl group can be alkylated to give oil soluble ether derivatives such as artemether \& arteether - Esterification of the hydroxyl with succinic acid gives the water soluble derivative, artesunate SAR Of Artemisinin

- Studies of artemisinin analogues such as deoxy artemisinin which do not contain the Endoperoxide Bridge showed vastly reduced biological activity.

\section{Quinine \& Related Compounds:}


The bark of the cinchona tree contains antimalarial compounds, most notably the highly fluorescent compound, quinine.

- The bark of the cinchona tree, if made into an aqueous solution was able to treat most cases of malaria.

- The active principle quinine was first isolated from the bark during the early 19th century.

- Quinine is the compound that contributes to the bitter taste of tonic water.

\section{4-aminoquinolines}

Increasing concern about cinchona supplies and the desire to find quinine alternatives with reduced side effects led to a massive search for novel antimalarials.

- Chloroquine was one of the drugs successfully developed. The drug was first used during the 1950s.

- Chloroquine is effective against erythrocytic forms of the Plasmodium parasite. Like chloroquine, the drugs amodiaquine and hydroxychloroquine belong to a class of quinine analogues called 4-aminoquinolines.4-aminoquinoline.

\section{Drug designed of Chloroquine as prototype drug:}

It consists of 4- aminoquinoline pharmacophore.

- The structural analogues of chloroquine have been designed in such a way that it will show more drug likeness score than the prototype molecule but having the same pharmacophore essential for the antimalarial activity. The side chain present at 4 position of chloroquine has been modified with alteration of halogen atom in some cases at position 8 to get increased drug likeness score.

- In case of designed molecules the chlorine molecule at position 8 has been replaced by $\mathrm{F}$ atom to increase the drug likeness score than the prototype molecule chloroquine.

- The position of R1 and R2 in the 4- amino quinolone ring are modified in these designed molecules to get increased drug likeness score.8-aminoquinolines

- Drugs in this group have amino group at position 8 of quinoline ring

- Such drugs have OCH3 group at position 6

- Pamaquine, primaquine, and tafenoquine are antimalarial drugs that belong to a family named 8- aminoquinolines.

- Pamaquine is closely related to primaquine. - Compared to primaquine, pamaquine is more toxic and less effacacious. 
- Tafenoquine is currently in late clinical trials. When side chain is introduced at amino group antimalarial activity is intensified - It causes hemolysis of RBCs Diethyl amino pentyl side chain Pamaquine

- It contains tertiary amino group and when it is converted into primary amino group the compound is called primaquine, which is - Less toxic - Well tolerated It is the most commonly used agent in this group in the treatment of malaria Primaquine

- $\mathrm{OCH} 3$ is not necessary for antimalarial activity but when replaced by OC2H5 the compound became - less active - Toxic in nature

- $\mathrm{OCH} 3$ when replaced by $\mathrm{CH} 3$ the compound become inactive

- Introduction of halogens increases toxicity.

- Presence of quinoline ring is necessary for antimalarial activity. When pyridine ring is converted to piperidine (saturated) the compound became inactive

- Pentyl side chain gives maximum activity, increase or decrease of chain result is reduction of activity.

- The branched side chain when converted into straight chain pentaquine is obtained - It has less antimalarial activity as compared to both pamaquine and primaquine.

\section{Antifolate combination drugs:}

These drugs are various combinations of dihydroolate-reductase inhibitors (proguanil, chlorproguanil, pyrimethamine, and trimethoprim) and sulfa drugs (dapsone, sulfalene, sulfamethoxazole, sulfadoxine, and others). Although these drugs have antimalarial activity when used alone, parasitological resistance can develop rapidly. When used in combination, they produce a synergistic effect on the parasite and can be effective even in the presence of resistance to the individual components. Typical combinations include sulfadoxine/ pyrimethamine (SP or Fansidar1), sulfalene pyrimethamine (metakelfin), and sulfamethoxazoletrimethoprim (co-trimoxazole). A new antifolate combination drug is currently being tested in Africa.

This drug, a combination of chlorproguanil and dapsone, also known as LapDap, has a much more potent synergistic effect on malaria than existing drugs such as SP. Benefits of this combination include

1) a greater cure rate, even in areas currently experiencing some level of SP resistance,

2) a lower likelihood of resistance developing because of a more advantageous pharmacokinetic and pharmacodynamic profile, and

3) Probable low cost (currently estimated at less than US\$ 1 per adult treatment course) 


\section{Combination therapy with antimalarials:}

The use of two antimalarials simultaneously, especially when the antimalarials have different mechanisms of action, has the potential for inhibiting the development of resistance to either of the components. The efficacy of a combination of a 4-aminoquinoline drug (either chloroquine or amodiaquine) with sulfadoxine/pyrimethamine has been reviewed (43). The results of this review suggested that the addition of either chloroquine or amodiaquine to SP marginally improved parasitological clearance (compared with SP alone). The studies reviewed were mostly done in areas and at times when both SP and chloroquine/amodiaquine retained a fair amount of efficacy, and it is not clear from these studies how well such a combination would act in areas where one of the components was significantly compromised. Additionally, to date, there are no data to suggest whether this slightly improved clearance would translate into prolonged useful life span for either drug. Another combination therapy approach, combining an artemisinin derivative with other, longer half-life antimalarials.

\section{Current status of drug-resistant malaria:}

Resistance to antimalarial drugs has been described for two of the four species of malaria parasite that naturally infect humans, P. falciparum and P. vivax. P. falciparum has developed resistance to nearly all antimalarials in current use, although the geographical distribution of resistance to any single antimalarial drug varies greatly. P. vivax infection acquired in some areas has been shown to be resistant to chloroquine and/or primaquine $(44,45)$. Chloroquineresistant P. falciparum malaria.

\section{CONCLUSION:}

Because of the realities of health care infrastructure and the influence of the private sector, approaches to malaria therapy, especially in sub-Saharan Africa, will probably favour increased access to drugs (and, therefore, loss of control over how they are used) over restricted access (and, therefore, more control over how they are used). If this proves to be true, while only minor advances against antimalarial drug. A more cautious approach would be to avoid placing too much faith in future scientific advances and technology and to invest in methods to improve the way people and antimalarial drugs interact in an environment of essentially uncontrolled use. The objective of this investment would be to prolong the useful life span of drugs enough to increase the likelihood that new drugs or other methods of malaria control will indeed be developed and implemented.

REFERENCE: 
1. Foster SD. Pricing, distribution, and use of antimalarial drugs. Bulletin of the World Health Organization 1991; 69:349-363.

2. Ridley RG. Plasmodium: Drug discovery and development - an industrial perspective. Experimental Parasitology 1997;87:293-304.

3. World Health Organization. World malaria situation in 1993, part I. Weekly Epidemiological Record 1996; 71:17-22.

4. Nabarro DN, Talyer EM. The "Roll Back Malaria" campaign. Science 1998;280;20672068.

5. Nchinda TC. Malaria: A reemerging disease in Africa. Emerging Infectious Diseases 1998;4:398-403.

6. World Health Organization. MMV comes of age. TDR News 1999; No. 60:6.

7. Snow RW et al. Estimating mortality, morbidity and disability due to malaria among Africa's non-pregnant population. Bulletin of the World Health Organization 1999;77:624-640.

8. Foster S, Phillips M. Economics and its contribution to the fight against malaria. Annals of Tropical Medicine \& Parasitology 1998;92:391-398.

9. Knudsen AB, Slooff R. Vector-borne disease problems in rapid urbanization: new approaches to vector control. Bulletin of the World Health Organization 1992;70:1-6.

10. Jonkman A et al. Cost-saving through microscopy based versus presumptive diagnosis of malaria in adult outpatients in Malawi. Bulletin of the World Health Organization 1995;73:223-227.

11. Barat L et al. Does the availability of blood slide microscopy for malaria at health centers improve the management of persons with fever in Zambia? American Journal of Tropical Medicine and Hygiene 1999;60;1024-1030.

12. Levine RA, Wardlaw SC, Patton CL. Detection of haematoparasites using Quantitative Buffy Coat analysis tubes. Parasitology Today 1989;5:132-133.

13. Tharavanij S. New developments in malaria diagnostic techniques. Southeast Asian Journal of Tropical Medicine \& Public Health 1990;21:3-16.

14. Rickman LS et al. Rapid diagnosis of malaria by acridine orange staining of centrifuged parasites. Lancet 1989;1:68-71.

15. Olivar $\mathrm{M}$ et al. Presumptive diagnosis of malaria re-25 WHO/CDS/CSR/DRS/2001.4 Drug Resistance In Malaria 
16. Redd SC et al. Usefulness of clinical case-definitions in guiding therapy for African children with malaria or pneumonia. Lancet 1992;340:1140-1143.

17. Smith $\mathrm{T}$ et al. Attributable fraction estimates and case definitions for malaria in endemic areas. Statistics In Medicine 1994;13:2345-2358.

18. World Health Organization. Integrated Management of Childhood Illnesses Adaptation Guide. Part 2. C. Technical basis for adapting clinical guidelines, feeding recommendations, and local terms. Working Draft Version 3. Division of Child Health and Development, World Health Organization, 1997. pp. 49-51.

19. Mackey LJ et al. Diagnosis of Plasmodium falciparum infection in man: detection of parasite antigens by ELISA. Bulletin of the World Health Organization 1982;60:69-75.

20. Fortier B et al. Enzyme immunoassay for detection of antigen in acute Plasmodium falciparum malaria. European Journal of Clinical Microbiology 1987;6:596-598.

21. Khusmith $\mathrm{S}$ et al. Two-site immunoradiometric assay for detection of Plasmodium falciparum antigen in blood using monoclonal and polyclonal antibodies. Journal of Clinical Microbiology 1987; 25:1467-1471.

22. Craig MH, Sharp BL. Comparative evaluation of four techniques for the diagnosis of Plasmodium falciparum infections. Transactions of the Royal Society of Tropical Medicine and Hygiene 1997; 91:279-282.

23. World Health Organization. A rapid dipstick antigen capture assay for the diagnosis of falciparum malaria. WHO informal consultation on recent advances in diagnostic techniques and vaccines for malaria. Bulletin of the World Health Organization 1996; 74:47-54.

24. Makler MT, Palmer CJ, Ager AL. A review of practical techniques for the diagnosis of malaria. Annals of Tropical Medicine \& Parasitology 1998; 92:419-433.

25. Piper $\mathrm{R}$ et al. Immunocapture diagnostic assays for malaria using Plasmodium lactate dehydrogenase (pLDH). American Journal of Tropical Medicine and Hygiene 1999; 60:109-118

26. Palmer CJ et al. Field evaluation of the OptiMAL7 rapid malaria diagnostic test during anti-malarial therapy in Guyana. Transactions of the Royal Society of Tropical Medicine \& Hygiene 1999; 93:517-518.

27. Beck HP. How does molecular epidemiology help to understand malaria? Tropical Medicine and International Health 1999;4:1-3. 
28. Freeman $\mathbf{J}$ et al. Effect of chemotherapy on malaria transmission among Yanomami Amerindians: simulated consequences of placebo treatment. American Journal of Tropical Medicine and Hygiene 1999; 60:774-780.

29. Watkins WM et al. The efficacy of antifolate antimalarial combinations in Africa: a predictive model based on pharmacodynamic and pharmacokinetic analyses. Parasitology Today 1997; 13:459-464.

30. Kremsner PG et al. Clindamycin treatment of falciparum malaria in Brazil. Journal of Antimicrobial Chemotherapy 1989; 23:275-281.

31. Kremsner PG et al. Clindamycin in combination with chloroquine or quinine is an effective therapy for uncomplicated Plasmodium falciparum malaria in children from Gabon. The Journal of Infectious Diseases 1994;169:467-470.

32. White NJ et al. Averting a malaria disaster. Lancet 1999; 353:1965-1967.

33. Price RN et al. Effects of artemisinin derivatives on malaria transmissiblity. Lancet 1996; 347:1654- 1658 .

34. Nosten F et al. Cardiac effects of antimalarial treatment with halofantrine. Lancet 1993; 341:1054- 1056.

35. Looareesuwan $\mathrm{S}$ et al. Clinical studies of atovaquone, alone or in combination with other antimalarial drugs for the treatment of acute uncomplicated malaria in Thailand. American Journal of Tropical Medicine and Hygiene 1996; 54:62-66.

36. Radloff PD et al. Atovaquone plus proguanil is an effective treatment for Plasmodium ovale and P. malariae malaria. Transactions of the Royal Society of Tropical Medicine \& Hygiene 1996;90:682

37. Bloland PB et al. Malarone-donation programme in Africa. Lancet 1997;350:1624-1625.

38. Foege WH. Malarone-donation programme. Lancet 1997; 350:1628-1629.

39. Ringwald P, Bickii J, Basco L. Randomised trial of pyronaridine versus chloroquine for acute uncomplicated falciparum malaria in Africa. Lancet 1996; 347:24-27.

40. Looareesuwan S et al. Pyronaridine. Lancet 1996; 347:1189-1190.

41. Olliaro PL, Trigg PI. Status of antimalarial drugs under development. Bulletin of the World Health Organization 1995;73:565-571.

42. van Vugt $M$ et al. Efficacy of six doses of artemetherlumefantrine (Benflumetol) in multidrug-resistant Plasmodium falciparum malaria. American Journal of Tropical Medicine and Hygiene 1999; 60:936-942. 
43. McIntosh HM, Greenwood BM. Chloroquine or amodiaquine combined with sulfadoxine-pyrimethamine as a treatment for uncomplicated malaria.

AJPHR is

Peer-reviewed

monthly

Rapid publication

Submit your next manuscript at

editor@ajphr.com / editor.ajphr@gmail.com 